\title{
Effect of position and positive pressure ventilation on functional residual capacity in morbidly obese patients: a randomized trial Effet de la position et de la ventilation en pression positive sur la capacité résiduelle fonctionnelle chez les patients obèses morbides : une étude randomisée
}

\author{
Etienne J. Couture, MD, FRCPC • Steeve Provencher, MD, MSc, FRCPC • \\ Jacques Somma, B. Eng, MD, FRCPC • François Lellouche, MD, PhD • \\ Simon Marceau, MD, FRCPC • Jean S. Bussières, MD, FRCPC
}

Received: 31 July 2017/Revised: 8 December 2017 / Accepted: 9 December 2017/Published online: 18 January 2018

(C) Canadian Anesthesiologists' Society 2018

\begin{abstract}
Purpose In morbidly obese patients, the position and ventilation strategy used during pre-oxygenation influence the safe non-hypoxic apnea time and the functional residual capacity (FRC). In awake morbidly obese volunteers, we hypothesized that the FRC would be higher after a five-minute period of positive pressure ventilation compared with spontaneous ventilation at zero inspiratory pressure.

Methods Using a prospective crossover randomized trial design, obese subjects underwent, in a randomized order, a combination of one of three positions, supine $(S)$, beach
\end{abstract}

E. J. Couture, MD, FRCPC

Department of Anesthesiology and Critical Care, Laval

University, Québec, Canada

S. Provencher, MD, MSc, FRCPC

Department of Pneumology, Institut Universitaire de Cardiologie et de Pneumologie de Québec, Université Laval, Québec City, QC, Canada

J. Somma, B. Eng, MD, FRCPC · J. S. Bussières, MD, FRCPC ( $\square)$

Department of Anesthesiology, Institut Universitaire de Cardiologie et de Pneumologie de Québec, Université Laval, 2725, Chemin Sainte-Foy, Québec City, QC G1V 4G5, Canada e-mail: jbuss@criucpq.ulaval.ca

F. Lellouche, MD, $\mathrm{PhD}$

Department of Critical Care, Institut Universitaire de

Cardiologie et de Pneumologie de Québec, Université Laval,

Québec City, QC, Canada

S. Marceau, MD, FRCPC

Department of Bariatric Surgery, Institut Universitaire de Cardiologie et de Pneumologie de Québec, Université Laval, Québec City, QC, Canada chair $(B C)$, and reverse Trendelenburg $(R T)$, and one of two ventilation strategies, spontaneous ventilation at zero inspiratory pressure (ZEEP-SV) or with positive pressure $(\mathrm{PP}-\mathrm{SV})$ set to an inspiratory pressure of $8 \mathrm{cmH}_{2} \mathrm{O}$, positive end-expiratory pressure of $10 \mathrm{cmH}_{2} \mathrm{O}$, and fraction of inspired oxygen of 0.21 .

Results Seventeen obese volunteers with a mean (standard deviation; SD) body mass index of 50 (8) $\mathrm{kg} \cdot \mathrm{m}^{-2}$ were included. Mean (SD) FRC in the three positions ( $S, B C$, $R T)$ was significantly higher using PP-SV compared with ZEEP-SV [2571 (477) vs 2215 (481) $m L$, respectively; mean difference, 356; 95\% confidence interval (CI), 209 to 502; $P<0.001$ ]. Mean (SD) FRC was significantly higher in the RT compared with BC position [2483 (521) vs 2338 (469) $\mathrm{mL}$, respectively; mean difference, 145 ; $95 \%$ CI, 31 to 404; $P=0.01$ ], while there was no difference between $S$ and $B C$ [2359 (519) $m L$ vs 2338 (469) $m L$, respectively; mean difference, 21; $95 \% C I$, -93 to 135; $P=0.89$ ].

Conclusion In awake morbidly obese volunteers, an increase in the FRC is observed when spontaneous ventilation at zero inspiratory pressure is switched to positive pressure. Compared with $S$ positioning, the $B C$ position had no measurable impact on the FRC. The RT position resulted in an optimal FRC.

Trial registration clinicaltrials.gov (NCT02121808). Registered 24 April 2014.

Résumé

Objectif Chez les patients obèses morbides, la position et la stratégie de ventilation utilisées pendant la pré-oxygénation ont un impact sur la durée d'apnée sans désaturation et la capacité résiduelle fonctionnelle (CRF). Avec la participation de volontaires obèses morbides 
éveillés, nous avons émis l'hypothèse que la CRF serait plus élevée après cinq minutes de ventilation en pression positive par rapport à une ventilation spontanée sans pression inspiratoire.

Méthode En se fondant sur une méthodologie d'étude randomisée croisée prospective, des volontaires obèses ont été soumis, de façon aléatoire, à une combinaison d'une de trois positions, soit la position allongée sur le dos (A), la position semi-assise (SA) et la position proclive (anti-Trendelenburg) ( $P$ ), et de l'une de deux stratégies de ventilation, soit une ventilation spontanée sans pression inspiratoire (VS-0) ou avec une pression positive (VS-PP) établie à une pression inspiratoire égale à $8 \mathrm{~cm} \mathrm{H}_{2} \mathrm{O}$, une pression positive télé-expiratoire de $10 \mathrm{~cm} \mathrm{H}_{2} \mathrm{O}$, et une concentration d'oxygène inspiré de 0,21.

Résultats Dix-sept volontaires obèses avec un indice de masse corporel moyen (écart type; ÉT) de 50 (8) $\mathrm{kg} \cdot \mathrm{m}^{-2}$ ont été inclus dans notre étude. La CRF moyenne (ÉT) dans les trois positions $(A, S A, P)$ était significativement plus élevée lorsqu'on a utilisé une VS-PP par rapport à une VS-O [2571 (477) vs 2215 (481) mL, respectivement; différence moyenne, 356; intervalle de confiance (IC) 95 \%, 209 à 502; $P<0,001]$. La CRF moyenne (ÉT) était significativement plus élevée dans la position $P$ par rapport à la position SA [2483 (521) vs 2338 (469) $\mathrm{mL}$, respectivement; différence moyenne, 145; IC $95 \%, 31$ à 404; $P=0,01]$, alors qu'aucune différence n'a été observée entre les positions A et SA [2359 (519) mL vs 2338 (469) $m L$, respectivement; différence moyenne, 21; IC $95 \%,-93$ à 135; $P=0,89]$.

Conclusion Chez des volontaires obèses morbides éveillés, on observe une augmentation de la CRF lorsqu'on passe d'une ventilation spontanée sans pression inspiratoire à une ventilation spontanée en pression positive. Par rapport à un positionnement allongé, la position semi-assise n'a pas eu d'impact mesurable sur la CRF. La position proclive a eu pour résultat une CRF optimale.
Enregistrement
de
l'étude clinicaltrials.gov
(NCT02121808). Enregistrée le 24 avril 2014.

Complications during intubation are a major contributing factor to morbidity after anesthesia. ${ }^{1}$ Furthermore, patients with morbid obesity have a four-fold increase in airwayrelated complications compared with non-obese patients. ${ }^{2,3}$ Alterations in respiratory mechanics and increased oxygen consumption in patients with a body mass index (BMI) $>$ $40 \mathrm{~kg} \cdot \mathrm{m}^{-2}$ contribute to a greater risk of oxygen desaturation with the induction of general anesthesia. ${ }^{4}$ Intra-abdominal mass and thoracic wall adiposity create a restriction of diaphragm displacement and reduce chest wall compliance. The resultant restrictive pattern of respiratory mechanics is associated with a marked reduction in functional residual capacity (FRC) and an increase in airway resistance. ${ }^{4,5}$ Moreover, obese patients are also susceptible to atelectasis, leading to further ventilation/perfusion mismatch and hypoxemia. ${ }^{6}$

Pre-oxygenation prior to anesthetic induction creates a safety margin by increasing patient oxygen reserves. ${ }^{7}$ As obesity may be associated with an increased risk of difficult intubation, ${ }^{8}$ ensuring adequate pre-oxygenation is important to prolong the safe non-hypoxic apnea time via increasing the oxygen content in the FRC before the onset of apnea. ${ }^{9}$ Given that morbidly obese patients have markedly reduced FRC, maneuvers that increase patient FRC can play a crucial role in optimizing oxygen stores and successful pre-oxygenation. ${ }^{10}$ These maneuvers include both optimal patient positioning ${ }^{11}$ and ventilation strategies. ${ }^{12,13}$ Head-elevated positions using ramped and beach-chair (BC) positions are currently recommended and can also be used to optimize the laryngoscopic view during tracheal intubation. ${ }^{14}$ Nevertheless, the literature is mixed with respect to whether these positions are associated with improving FRC. Understanding the impact of different combinations of patient position and ventilator strategy on FRC would allow clinicians to choose the best combination to increase the non-hypoxemic apnea time for intubation.

Accordingly, this present study was designed to evaluate the effect of three different positions and two different spontaneous ventilation strategies on FRC in morbidly obese volunteers during pre-oxygenation ventilation. In awake morbidly obese volunteers, we hypothesized that FRC would be greater after a five-minute period of positive pressure ventilation compared with spontaneous ventilation at zero inspiratory positive pressure. We further hypothesized that FRC would be higher, irrespective of ventilation strategy, when undertaken in various headelevated positions (i.e., BC and reverse Trendelenburg [RT] positions) compared with a supine (S) position.

\section{Methods}

Study population

Twenty volunteers $\geq 21 \mathrm{yr}$ with a BMI of $40-80 \mathrm{~kg} \cdot \mathrm{m}^{-2}$ and a waist circumference $>115 \mathrm{~cm}$ for females and $>130$ $\mathrm{cm}$ for males were included in this randomized, prospective, crossover study. The institutional review board and ethics committee of our institution approved the study protocol (14 April 2014; CER \#21054) and written informed consent was obtained from all volunteers. Subjects were recruited from the preoperative bariatric 
surgery clinic of the Institut universitaire de cardiologie et de pneumologie de Québec between 24 April 2014 and 26 June 2014. Patients with major craniofacial abnormalities, claustrophobia, asthma, chronic obstructive pulmonary disease (defined as forced expiratory volume in one second $<80 \%$ of predicted value), severe cardiac failure (defined as New York Heart Association classification $>$ III), pregnancy, active tobacco use, and intolerance to noninvasive positive pressure ventilation (NIPPV) through a facemask were excluded.

\section{Study design and procedures}

The design of this randomized, double-blind, crossover study is shown in Fig. 1. Patients fasted for eight hours and received oral ranitidine $150 \mathrm{mg}$ before the beginning of the intervention, which was conducted in the respiratory physiology laboratory of our institution. Anthropometric measures were taken and subjects (wearing only a hospital gown) were positioned on an operating table (Universal operating table, Alphamaxx; Maquet, Rastatt, Germany) after which pulse oximetry, five-lead electrocardiography, and non-invasive blood pressure monitoring were applied. A retention strap was secured $10 \mathrm{~cm}$ above the knee (with no other restraints used). Footplates were used with the RT position. After positioning, vital signs and vital capacity were measured. Baseline FRC corresponds to spontaneous ventilation without positive pressure support (ZEEP-SV) in the $\mathrm{S}$ position.

The intervention began with one of the six combinations of three different positions and two modes of ventilation in a randomly determined manner. The positions were $\mathrm{S}$ (back inclination $0^{\circ}$ ), $\mathrm{BC}$ (back inclination $25^{\circ}$ with horizontal legs), and RT (table inclination $25^{\circ}$ ). Angles of the legback articulation of the operating table were set and confirmed with a digital angle level (Digital Angle Level; ROK, Burnaby, BC, Canada). The ventilation modes were ZEEP-SV or spontaneous ventilation with positive pressure support (PP-SV). A computerized randomization sequence was created that included various combinations of patient position and ventilation strategy. These were placed in sealed envelopes that were then opened by a research assistant after the volunteer had been positioned and was ready to start the experimental sequence. The volunteers were allowed to pause for $20 \mathrm{~min}$ after each position/ ventilation period during which it was possible for them to stand-up, walk, and sit without the presence of a mouthpiece or monitoring.

While in the ZEEP-SV mode, the study subjects were coached to keep the same tidal volume during the whole experiment. Spontaneous ventilation with positive pressure was provided by a mechanical ventilator (Puritan Bennett 840; Covidien, Mansfield, MA, USA) set to an inspiratory

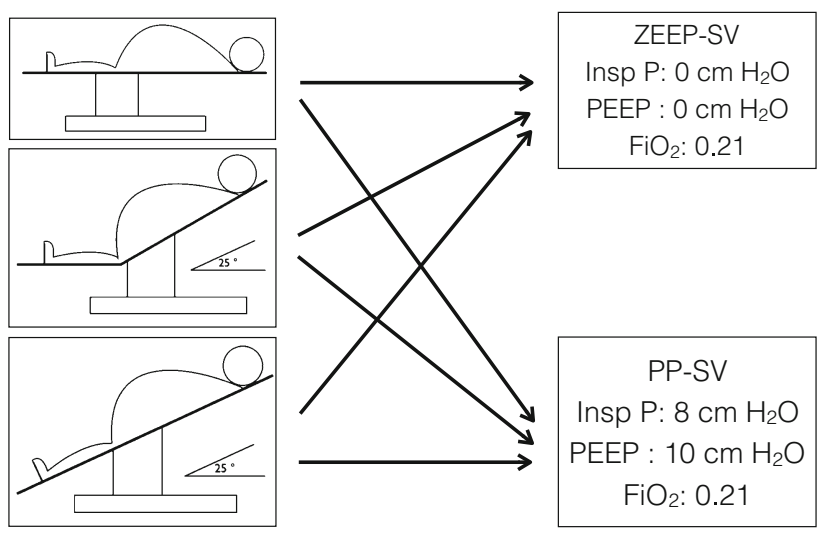

Fig. 1 Design of the randomized, double-blind crossover study. $\mathrm{F}_{1} \mathrm{O}_{2}$ $=$ fraction of inspired oxygen, InspP $=$ inspiratory pressure; $\mathrm{PEEP}=$ positive end-expiratory pressure; $\mathrm{PP}-\mathrm{SV}=$ spontaneous ventilation with positive pressure; ZEEP-SV = spontaneous ventilation at zero inspiratory pressure

pressure of $8 \mathrm{cmH}_{2} \mathrm{O}$ above a positive end-expiratory pressure of $10 \mathrm{cmH}_{2} \mathrm{O}$. During positive pressure ventilation, the subjects were connected to the breathing circuit using a soft snorkel-type mouthpiece (Airlife adult flexible mouthpiece; CareFusion, San Diego, CA, USA) with a nose clip. In both ZEEP-SZ and PP-SV conditions, a fraction of inspired oxygen $\left(\mathrm{F}_{\mathrm{I}} \mathrm{O}_{2}\right)$ of 0.21 was used throughout to minimize the risk of denitrogenation atelectasis and its confounding effects on FRC measurements. At the end of each position/ventilation combination period, oxygen saturation, respiratory frequency, and tidal volume were recorded after which an FRC measurement was made.

Functional residual capacity measurement

The FRC was measured using a helium dilution method. At the end of every five-minute study period, the ventilation interface was switched from ZEEP-SV or PP-SV to a closed system synchronously at the end of a normal expiratory phase to allow for the helium dilution FRC measurement (HypAir; Medisoft, Sorinnes, Belgium) according to the American Thoracic Society/European Respiratory Society guidelines. ${ }^{15}$ Vital capacity and oxygen consumption were obtained once the helium concentration was stable and the FRC obtained. The gas mixture used for the FRC measurements was composed of oxygen $60 \%$, helium $14 \%$, and air added for a constant volume of 7 L. Functional residual capacity measurement was obtained when the system reached a steady state with the respiratory system of the patient $(<0.02 \%$ helium concentration variation over a $30-\mathrm{sec}$ period). Equilibrium was generally obtained after three minutes. 


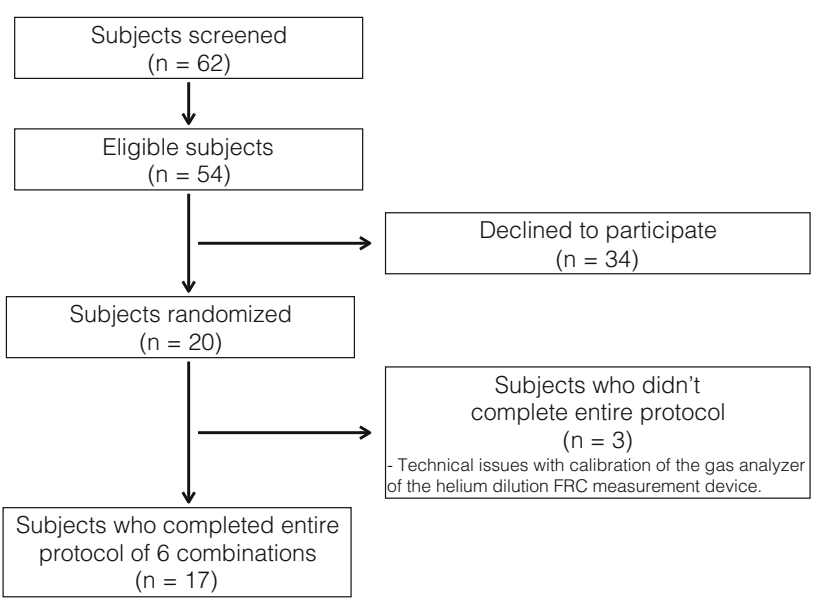

Fig. 2 Consort flowchart

Table 1 Demographic data

\begin{tabular}{ll}
\hline Age $(\mathrm{yr})$ & $40(10)$ \\
\hline Gender $(\mathrm{M} / \mathrm{F})$ & $5 / 12$ \\
Weight $(\mathrm{kg})$ & $144(30)$ \\
Ideal weight $(\mathrm{kg})^{*}$ & $62(9)$ \\
Height $(\mathrm{cm})$ & $169(8)$ \\
BMI $\left(\mathrm{kg} \cdot \mathrm{m}^{-2}\right)$ & $50(8)$ \\
Waist circumference $(\mathrm{cm})$ & $143(17)$ \\
Hip circumference $(\mathrm{cm})$ & $154(18)$ \\
Waist:hip ratio & $0.93(0.09)$ \\
Neck circumference $(\mathrm{cm})$ & $46(6)$ \\
CPAP users & 8 \\
STOP-BANG score & $3.3(1.3)$ \\
\hline
\end{tabular}

Results are presented as mean (standard deviation). BMI = body mass index; $\mathrm{CPAP}=$ continuous positive airway pressure

*The formula used for ideal weight is as follows:

Female, $45.5+0.91$ [height $(\mathrm{cm})-152.4$ ]

Male, $50+0.91$ height $(\mathrm{cm})-152.4]$

\section{Statistical analysis}

The primary endpoint of the study was the absolute difference in the mean FRC between the two ventilation strategies irrespective of the position used. The secondary endpoint was the absolute differences in the mean FRC among the three tested positions irrespective of the ventilation strategy used. As there was no study that had evaluated the modification of FRC after a brief period of NIPPV, the sample size calculation was based on a previous publication showing a mean (standard deviation; $\mathrm{SD}$ ) increase of non-hypoxemic apnea time of 61(43) sec with the use of NIPPV during pre-oxygenation. ${ }^{16}$ As an increase in non-hypoxemic apnea time is the consequence of an increase in FRC, an increase of $61 \mathrm{sec}$ would thus correspond to an estimated FRC increase of $355 \mathrm{~mL}$ based on an approximate $140 \mathrm{~kg}$ body weight and oxygen consumption calculation of $3.5 \mathrm{~mL} \cdot \mathrm{kg}^{-1} \cdot \mathrm{min}^{-1}$ for the first $70 \mathrm{~kg}$ and $1.5 \mathrm{~mL} \cdot \mathrm{kg}^{-1} \cdot \mathrm{min}^{-1}$ for every additional $\mathrm{kg} .{ }^{17}$ Considering a mean (SD) baseline FRC of 1550 (340) mL and an increase of $355 \mathrm{~mL}$ in FRC, 15 patients were needed to obtain an alpha error of $5 \%$ and a power of $80 \%$. We increased the sample size to 20 patients because of possible anticipated subject withdrawal and/or randomization failure. $^{16,18}$

Data were expressed as mean (SD). For each subject, comparisons between the two ventilation modes and three patient positions were performed using a mixed linear model with a hierarchical structure. Subjects were considered independent in their measurements. Withinsubject values were considered as correlated measurements. Comparisons between the two ventilation modes were the first level of repeated measurements. For each ventilation mode, three patient positions were performed on the table. This was defined as a second level of repeated measurements. The statistical linear model used a random intercept slope and a symmetric covariance structure for measurements from between ventilation modes as well as within. A more general covariance structure was investigated but was not significant compared with the symmetric covariance structure. Post hoc comparisons were performed using Tukey's technique. The multivariate normality assumption was verified with the Shapiro-Wilk test after a Cholesky factorization on residuals. The results were considered significant at $P<0.05$. All analyses were conducted using the statistical packages $\mathrm{R}$ v3.0.2 ( $\mathrm{R}$ Foundation for Statistical Computing, Vienna, Austria.) and SAS v9.4 (SAS Institute Inc., Cary, NC, USA).

\section{Results}

During the study period, 62 patients were screened for participation in the study. Seven were excluded because of asthma and one because of active tobacco smoking. Of the 54 patients eligible, 34 declined to consent. Thus, a total of 20 volunteers were randomized to participate in the protocol. Three subjects were withdrawn from the final analysis because they did not complete the entire protocol because of technical issues when calibrating the gas analyzer of the helium dilution FRC measurement device (Fig. 2). Baseline characteristics of the 17 subjects who completed the full protocol are shown in Table 1.

Under PP-SV (when combining the S, BC, and RT position groups), there was a $16 \%$ relative increase in mean (SD) FRC compared with ZEEP-SV [2571 (477) vs 2215 (481) $\mathrm{mL}$, respectively; mean difference, $356 \mathrm{~mL}$; $95 \%$ 
confidence interval (CI), 208 to 502; $P<0.001]$. When the ZEEP-SV and PP-SV groups were combined, the RT position was also associated with a small increase in FRC in comparison with both the $\mathrm{S}$ [2483 (521) vs 2359 (519) $\mathrm{mL}$; mean difference, $123 \mathrm{~mL} ; 95 \% \mathrm{CI}, 9$ to $237 ; P=0.03$ ] and $\mathrm{BC}$ positions [2483 (521) vs 2338 (469) $\mathrm{mL}$; mean difference, $145 \mathrm{~mL} ; 95 \% \mathrm{CI}, 31$ to $404 ; P=0.01]$. There was no difference in FRC between the $\mathrm{S}$ and $\mathrm{BC}$ positions [2359 (519) vs 2338 (469) mL; mean difference, $21 \mathrm{~mL}$; 95\% CI, -93 to $135 ; P=0.89$ ] (Table 2).

\section{Discussion}

This crossover trial studied the variation of FRC with six different combinations of position and ventilation strategies during a five-minute period in morbidly obese volunteers. The findings reveal that the spontaneous ventilation with positive pressure support strategy markedly increased FRC in awake, spontaneously breathing morbidly obese subjects. The present study also confirms the additional effects of the RT position on FRC values compared with the $\mathrm{BC}$ and $\mathrm{S}$ positions commonly used in anesthesiology practice for pre-oxygenation before anesthesia induction.

Morbid obesity represents a major challenge for anesthesiologists. The increased risk of hypoxemia and difficult airway management due to changes in orofacial anatomy may complicate intubation and eventually lead to morbidity and mortality in that specific population. The marked increase in anesthetic risk of obese patients has been mainly attributed to a substantial decrease in FRC precluding adequate pre-oxygenation. Indeed, there is a reverse linear relationship between BMI and FRC in obese patients with morbidly obese patients having a two-thirds reduction in FRC values compared with non-obese patients. This is a consequence of the marked reduction in expiratory reserve volume and, to a lesser extent, in residual volume compared with non-obese subjects. ${ }^{5}$
Previous studies documented changes in lung volume in obese patients resulted from a displacement of the diaphragm into the thorax under the influence of the abdominal fat, ${ }^{19}$ accounting for the decrease in lung and chest wall compliance. ${ }^{20}$

In an attempt to enhance the safe non-hypoxic apnea time, positive pressure spontaneous ventilation during the pre-oxygenation period has been proposed. Supporting this are previous studies documenting that the spontaneous positive pressure ventilation strategy during preoxygenation leads to increased non-hypoxic apnea time $^{16,21}$ and greater end-tidal oxygen values. ${ }^{22-24}$ The reason for this improvement in non-hypoxic apnea time, although having traditionally been attributed to increases in FRC, remains elusive. Consistent with these observations, the present study confirms that spontaneous ventilation with a positive pressure support strategy is favourable over the use of spontaneous ventilation without positive pressure support to increase FRC. This result likely explains the increase in the safe non-hypoxic apnea time reported in the literature. To our knowledge, these present data are the first demonstrating the effect of spontaneous positive pressure ventilation on FRC variation in awake morbidly obese subjects.

Importantly, changes in FRC are dependent on body position, ${ }^{25}$ especially in the obese population. ${ }^{18}$ As a result, the current literature recommends to maintain a high FRC to body weight ratio. ${ }^{7}$ Nevertheless, the effect of body position on FRC is likely variable according to the specific positions and patient characteristics. Indeed, in the obese, there is an increase in FRC when subjects are switched from the $\mathrm{S}$ to $90^{\circ}$ sitting position. ${ }^{18,25,26}$ Consistently, we also observed that FRC was higher in the RT compared with $\mathrm{S}$ positions. Similar results have been published by comparing the FRC difference between the $30^{\circ} \mathrm{BC}$ and $\mathrm{S}$ position in a population of full-term pregnant women. ${ }^{27}$ Leg position in the RT might help to unload the diaphragm and increase FRC by accentuating excursion through the ventilation trial. Conversely, the present results differ from

Table 2 Functional residual capacity with changes in ventilation and position

\begin{tabular}{lllll}
\hline & Supine & Beach chair & Reverse Trendelenburg & Ventilation strategy specific \\
\hline ZEEP-SV & $2145(438) \mathrm{mL}$ & $2219(477) \mathrm{mL}$ & $2282(501) \mathrm{mL}$ & $2215(481)^{\mathrm{d}} \mathrm{mL}$ \\
PP-SV & $2573(515) \mathrm{mL}$ & $2456(442) \mathrm{mL}$ & $2684(473) \mathrm{mL}$ & $2571(477)^{\mathrm{d}} \mathrm{mL}$ \\
Position specific & $2359(519)^{\mathrm{ab}} \mathrm{mL}$ & $2338(469)^{\mathrm{ac}} \mathrm{mL}$ & $2483(521)^{\mathrm{bc}} \mathrm{mL}$ & - \\
\hline
\end{tabular}

$\mathrm{ZEEP}-\mathrm{SV}=$ spontaneous ventilation without positive pressure; PP-SV = spontaneous ventilation with positive pressure. Values are presented as mean (standard deviation)

${ }^{a} P=0.89$ between supine and beach chair

${ }^{\mathrm{b}} P=0.03$ between reverse Trendenenburg and supine

${ }^{c} P=0.01$ between reverse Trendelenburg and beach chair

${ }^{\mathrm{d}} P<0.001$ between ZEEP-SV and PP-SV 
those published by Benedik et al. that showed an absence of FRC variation between the $\mathrm{S}$ and $30^{\circ} \mathrm{RT}$ position in mildly to moderately obese subjects (BMI 30 to 39.9 $\left.\mathrm{kg} \cdot \mathrm{m}^{-2}\right) .{ }^{18}$ The morbid obesity observed in the present study population $\left[\right.$ mean $(\mathrm{SD}) \mathrm{BMI}=50(8) \mathrm{kg} \cdot \mathrm{m}^{-2}$; mean (SD) waist circumference $=143(17) \mathrm{cm}]$ might have accentuated the reduction of lung volumes and could explain the presence of a significant difference in FRC between the RT and the S position.

Head-elevated positions are commonly used for preoxygenation and induction of general anesthesia in the obese population though often for the purpose of optimizing the laryngoscopic view. ${ }^{28}$ As the oxygen reserves for the safety margin during airway management in lean patients are provided by the amount stored in the FRC during pre-oxygenation, ${ }^{29}$ increasing patient FRC during pre-oxygenation is expected to result in an increased safe non-hypoxic apnea time. We thus estimate that the impact of the various position/ventilation strategies on FRC is clinically relevant in this at-risk population. Not surprisingly, in morbidly obese patients, the use of a $\mathrm{BC}^{11,30}$ or RT position ${ }^{31}$ is associated with a longer safe non-hypoxic apnea time and higher partial pressure of $\mathrm{O}_{2}$ and end-tidal $\mathrm{O}_{2}\left(\mathrm{EtO}_{2}\right)$ after the pre-oxygenation period. Furthermore, Boyce et al. showed that there is an advantage to using an RT instead of BC position in terms of duration of safe non-hypoxic apnea time, oxygen desaturation, and time of recuperation of oxygen saturation following ventilation after intubation. Nevertheless, they did not use positive pressure ventilation and did not base the choice of the position on FRC values. ${ }^{31}$

The mean BMI of our study subjects represents that of morbid obesity, ${ }^{32}$ which corresponds to the type of patients that might need bariatric surgery or any other surgery and have to be pre-oxygenated with great care to limit the burden of possible airway complication.

The main limitation of our study is that FRC values were not correlated with the actual non-hypoxemic apnea time. Likewise, a $\mathrm{F}_{1} \mathrm{O}_{2}$ of 0.21 , which does not represent common clinical practice, was used through the experiment to minimize the risk of denitrogenation atelectasis and its confounding effects on repeated FRC measurements. Also, the effectiveness of the RT and positive pressure spontaneous ventilation was not correlated with the arterial blood gas values and $\mathrm{EtO}_{2}$ fraction. In addition, the FRC values obtained may not be directly related to the former clinically relevant non-hypoxemic apnea time for anesthesia providers. As a consequence, this study represents physiologic proof-of-concept findings to guide future studies to clinically evaluate the safe non-hypoxic apnea time comparing the best combination of positioning and ventilation strategy with the usual clinical approach.

\section{Conclusions}

The present study reveals that optimizing the ventilation strategy as well as patient position during a period of five minutes of simulation of pre-oxygenation can increase the FRC in morbidly obese patients. Irrespective of the position used, the spontaneous positive pressure ventilation strategy markedly increases FRC compared with spontaneous ventilation without positive pressure. Irrespective of the ventilation strategy used, the RT position further enhances FRC values compared with the $\mathrm{BC}$ position. The present results thus suggest that if clinicians have to make the choice between the headelevated position of BC and RT for a better laryngoscopic view, they should consider adopting the latter to also optimize the FRC. Future studies are needed to determine whether these physiologic changes in volunteers can be translated into changes of safe non-hypoxic apnea time in morbidly obese patients undergoing surgery.

Conflict of interest None declared.

Editorial responsibility This submission was handled by Dr. Hilary P. Grocott, Editor-in-Chief, Canadian Journal of Anesthesia.

Author contributions Etienne J. Couture made substantial contributions to conception and design, acquisition of data, interpretation of data, drafting the article, revising the article critically for important intellectual content, and final approval of the version to be published. Steeve Provencher and Jacques Somma made substantial contributions to conception and design, analysis and interpretation of data, revising the article critically for important intellectual content, and final approval of the version to be published. François Lellouche and Simon Marceau made substantial contributions to conception and design, revising the article critically for important intellectual content, and final approval of the version to be published. Jean S. Bussières made substantial contributions to conception and design, analysis and interpretation of data, drafting the article, and revising it critically for important intellectual content, and final approval of the version to be published.

Funding Research fund from Anesthesiology research team, Department of Anesthesiology, Institut Universitaire de Cardiologie et de Pneumologie de Québec - Université Laval.

\section{References}

1. Peterson GN, Domino KB, Caplan RA, Posner KL, Lee LA, Cheney $F W$. Management of the difficult airway: a closed claims analysis. Anesthesiology 2005; 103: 33-9.

2. Cook TM, Woodall N, Frerk C, Fourth National Audit Project. Major complications of airway management in the UK: results of the Fourth National Audit Project of the Royal College of Anaesthetists and the Difficult Airway Society. Part 1: anaesthesia. Br J Anaesth 2011; 106: 617-31.

3. Cook TM, Woodall N, Harper J, Benger J, Fourth National Audit Project. Major complications of airway management in the UK: results of the Fourth National Audit Project of the Royal College 
of Anaesthetists and the Difficult Airway Society. Part 2: intensive care and emergency departments. Br J Anaesth 2011; 106: 632-42.

4. Murphy C, Wong DT. Airway management and oxygenation in obese patients. Can J Anesth 2013; 60: 929-45.

5. Jones RL, Nzekwu MM. The effects of body mass index on lung volumes. Chest 2006; 130: 827-33.

6. Coussa M, Proietti S, Schnyder P, et al. Prevention of atelectasis formation during the induction of general anesthesia in morbidly obese patients. Anesth Analg 2004; 98: 1491-5.

7. Nimmagadda $U$, Salem MR, Crystal GJ. Preoxygenation: physiologic basis, benefits, and potential risks. Anesth Analg 2017; 124: 507-17.

8. Lundstrom LH, Moller AM, Rosenstock C, Astrup G, Wetterslev J. High body mass index is a weak predictor for difficult and failed tracheal intubation: a cohort study of 91,332 consecutive patients scheduled for direct laryngoscopy registered in the Danish Anesthesia Database. Anesthesiology 2009; 110: 266-74.

9. Farmery $A D$, Roe $P G$. A model to describe the rate of oxyhaemoglobin desaturation during apnoea. $\mathrm{Br} \mathrm{J}$ Anaesth 1996; 76: 284-91.

10. Shah U, Wong J, Wong DT, Chung F. Preoxygenation and intraoperative ventilation strategies in obese patients: a comprehensive review. Curr Opin Anaesthesiol 2016; 29: 109-18.

11. Dixon BJ, Dixon JB, Carden JR, et al. Preoxygenation is more effective in the 25 degrees head-up position than in the supine position in severely obese patients: a randomized controlled study. Anesthesiology 2005; 102: 1110-5; discussion 5A.

12. Valenza F, Vagginelli F, Tiby A, et al. Effects of the beach chair position, positive end-expiratory pressure, and pneumoperitoneum on respiratory function in morbidly obese patients during anesthesia and paralysis. Anesthesiology 2007; 107: 725-32.

13. Futier E, Constantin JM, Pelosi P, et al. Noninvasive ventilation and alveolar recruitment maneuver improve respiratory function during and after intubation of morbidly obese patients: a randomized controlled study. Anesthesiology 2011; 114: 135463.

14. Collins JS, Lemmens HJ, Brodsky JB, Brock-Utne JG, Levitan $R M$. Laryngoscopy and morbid obesity: a comparison of the "sniff" and "ramped" positions. Obes Surg 2004; 49: 1171-5.

15. Wanger J, Clausen JL, Coates A, et al. Standardisation of the measurement of lung volumes. Eur Respir J 2005; 26: 511-22.

16. Gander S, Frascarolo P, Suter M, Spahn DR, Magnusson L. Positive end-expiratory pressure during induction of general anesthesia increases duration of nonhypoxic apnea in morbidly obese patients. Anesth Analg 2005; 100: 580-4.

17. Porhomayon J, Papadakos P, Singh A, Nader ND. Alteration in respiratory physiology in obesity for anesthesia-critical care physician. HSR Proc Intensive Care Cardiovasc Anesth 2011; 3: 109-18.
18. Benedik PS, Baun MM, Keus L, et al. Effects of body position on resting lung volume in overweight and mildly to moderately obese subjects. Respir Care 2009; 54: 334-9.

19. Koenig SM. Pulmonary complications of obesity. Am J Med Sci 2001; 321: 249-79.

20. Pelosi P, Croci M, Ravagnan I, Vicardi P, Gattinoni L. Total respiratory system, lung, and chest wall mechanics in sedatedparalyzed postoperative morbidly obese patients. Chest 1996; 109: 144-51.

21. Cressey DM, Berthoud MC, Reilly CS. Effectiveness of continuous positive airway pressure to enhance pre-oxygenation in morbidly obese women. Anaesthesia 2001; 56: 680-4.

22. Delay JM, Sebbane M, Jung B, et al. The effectiveness of noninvasive positive pressure ventilation to enhance preoxygenation in morbidly obese patients: a randomized controlled study. Anesth Analg 2008; 107: 1707-13.

23. Tanoubi I, Drolet P, Fortier LP, Donati F. Inspiratory support versus spontaneous breathing during preoxygenation in healthy subjects. A randomized, double blind, cross-over trial. Ann Fr Anesth Reanim 2010; 29: 198-203.

24. Georgescu M, Tanoubi I, Fortier LP, Donati F, Drolet P. Efficacy of preoxygenation with non-invasive low positive pressure ventilation in obese patients: crossover physiological study (French). Ann Fr Anesth Reanim 2012; 31: e161-5.

25. Watson RA, Pride NB. Postural changes in lung volumes and respiratory resistance in subjects with obesity. J Appl Physiol 1985; 2005(98): 512-7.

26. Craig DB, Wahba WM, Don HF, Couture JG, Becklake MR. "Closing volume" and its relationship to gas exchange in seated and supine positions. J Appl Physiol 1971; 31: 717-21.

27. Hignett $R$, Fernando $R$, McGlennan A, et al. A randomized crossover study to determine the effect of a $30^{\circ}$ head-up versus a supine position on the functional residual capacity of term parturients. Anesth Analg 2011; 113: 1098-102.

28. Lee BJ, Kang JM, Kim DO. Laryngeal exposure during laryngoscopy is better in the $25^{\circ}$ back-up position than in the supine position. Br J Anaesth 2007; 99: 581-6.

29. Campbell IT, Beatty PC. Monitoring preoxygenation. $\mathrm{Br} \mathrm{J}$ Anaesth 1994; 72: 3-4.

30. Baraka AS, Hanna MT, Jabbour SI, et al. Preoxygenation of pregnant and nonpregnant women in the head-up versus supine position. Anesth Analg 1992; 75: 757-9.

31. Boyce JR, Ness T, Castroman P, Gleysteen JJ. A preliminary study of the optimal anesthesia positioning for the morbidly obese patient. Obes Surg 2003; 13: 4-9.

32. Anonymous. Clinical guidelines on the identification, evaluation, and treatment of overweight and obesity in adults - the evidence report. National Institutes of Health. Obes Res 1998; 6 Suppl 2: 51S-209S. 